\title{
The future of stem cells: Should we keep the "stem" and skip the "cells"?
}

\author{
Philippe Menasché, $\mathrm{MD}, \mathrm{PhD}$
}

\begin{abstract}
There is accumulating evidence that the cardioprotective effects of stem cells are predominantly mediated by the release of a blend of factors, possibly clustered into extracellular vesicles, which harness endogenous repair pathways. The clinical translation of this concept requires the identification of the cell-secreted signaling biomolecules and an appropriate transfer method. The study by Wei and colleagues has addressed these 2 requirements by showing that the epicardial delivery of a collagen patch loaded with the cardiokine follistatin-like 1 improved left ventricular function in animal models of myocardial infarction. Beyond the choice of the factor and its vehicle, these data may open a new therapeutic path whereby the functionalization of biomaterials by bioactive compounds could successfully substitute for the current cell transplantation-based strategy. (J Thorac Cardiovasc Surg 2016;152:345-9)
\end{abstract}

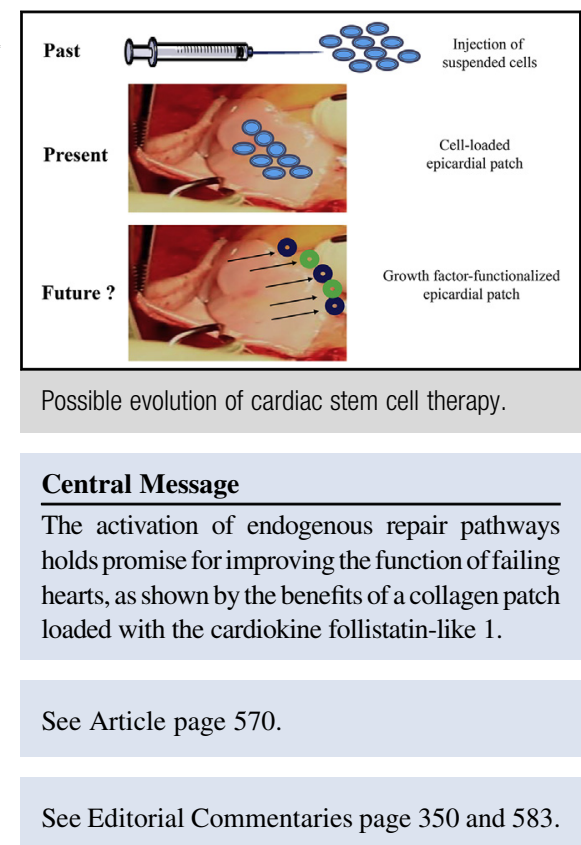

Editor's Note-This article is one of the first in our series that will highlight important new discoveries that may impact thoracic surgeons in the not too distant future. The Journal has recruited a number of Featured Editors to scan the literature to identify important new information that we believe will be of interest to our readers. Feature Editor Craig Smith identified this important article in Nature by Wei and colleagues that identified factors that stimulated endogenous cardiac stem cells to regenerate the heart after a myocardial infarction. The Editors invited Professor Philippe Menasche to write the Expert Opinion piece because of his experience with clinical trials of regenerative approaches. We hope that you enjoy this series and the contribution of Dr Menasche.

\section{Richard D. Weisel, MD \\ Editor}

From the Department of Cardiovascular Surgery, Hôpital Européen Georges Pompidou, University Paris Descartes, Sorbonne Paris Cité; and INSERM U 970, Paris, France.

Received for publication Feb 22, 2016; accepted for publication Feb 23, 2016; available ahead of print March 25, 2016.

Address for reprints: Philippe Menasché, MD, PhD, Department of Cardiovascular Surgery, Hôpital Européen Georges Pompidou, 20 Rue Leblanc, 75015 Paris, France (E-mail: philippe.menasche@aphp.fr).

$0022-5223 / \$ 36.00$

Copyright (c) 2016 by The American Association for Thoracic Surgery

http://dx.doi.org/10.1016/j.jtcvs.2016.02.058
In the September 24, 2015, issue of Nature, Wei and colleagues $^{1}$ reported that the epicardial delivery of a collagen patch loaded with the cardiokine follistatin-like 1 (Fstl-1) improves cardiac function in mouse and swine models of myocardial infarction. The premise of this study was that Fstl-1 is a regenerative factor that is normally present in uninjured hearts but is down-regulated after myocardial infarction, thereby supporting the concept of restoring it through its release from an epicardial bioengineered construct.

The beneficial effects of the Fstl-1-loaded patch were observed after permanent coronary artery ligation, with immediate patch application, and after occlusion/reperfusion; in the latter setting, the patch was applied according to a more clinically relevant scenario, ie, at a later time (1 week later). However, 2 methodologic features of the study protocol call for caution in extrapolating the results for use in clinical practice. First, the study was performed in primarily mouse hearts; and large animal data, although encouraging (reduced scar size and improvement in contractile recovery), are limited to 2 swine. Second, when cardiomyocytes at various developmental stages were tested for their response to Fstl-1, only those featuring an immature phenotype were found to proliferate. The precise identity of the Fslt-1-responsive cells could not be tracked precisely, which calls into question the applicability of Fsl-1 effects to heart failure patients. 


\section{Abbreviation and Acronym}

Fstl-1 = cardiokine follistatin-like 1

Despite these caveats, the data reported in this paper convey at least 3 clinically relevant messages. The first is that an appropriately selected factor can foster endogenous repair pathways, thereby contributing to improvement of the functional outcome of infarcted hearts. In this particular case, such an improvement seems to have been mediated by a combination of effects (reduction of fibrosis, stimulation of angiogenesis, host cardiomyocyte proliferation) induced by Fstl-1, a cardiokine located in epicardial cells and the levels of which are depleted after myocardial infarction. The fact that delivery of Fslt-1 by the patch improved postinfarction function is therefore consistent with the role of epicardium-derived signals in cardiac regeneration. ${ }^{2}$ For cardiac surgeons, the common observation of a thick layer of fibrosis overlaying the scarred myocardium can call into doubt the concept that local delivery of any factor can reverse the phenomenon, and convert an akinetic into a contractile area.

However, beyond the choice of a given compound, the relevance of this study lies in the evidence that exogenously induced paracrine signaling plays a major role in heart repair. Although mechanical unloading of the left ventricle is one intervention that can induce such paracrine effects, as suggested by the increase in the number of cycling cardiomyocytes in myocardial biopsies taken before and after placement of a left ventricular assist device, ${ }^{3}$ the main triggers of these effects that have been studied so far are stem cells. The consistent discrepancy between the fact that stem cells are quickly cleared from transplanted tissue, yet a functional benefit persists over time is strong evidence that these cells release paracrine factors that trigger endogenous cardioreparative pathways. ${ }^{4}$

The practical, yet unsettled, question is thus to assess which factor(s) is the most effective. Some investigators have reported successful results with intramyocardial injection of conditioned medium released by mesenchymal stem cells in animal models of myocardial infarction., ${ }^{5,6}$ Likewise, in the study by Wei and colleagues, ${ }^{1}$ the conditioned medium collected from cultured epicardial cells increased the proliferation of mouse embryonic stem cellderived cardiomyocytes. The drawback of this strategy is that conditioned medium contains large proteins, which may be unstable in vivo and are unlikely to cross cell membranes and permeate the cells. An alternate strategy consists of focusing on a single growth factor, which was the case in the study by Wei and colleagues ${ }^{1}$ after mass spectrophotometry of the epicardium-derived conditioned medium had identified Fstl-1 as the only protein (among 1596 peptide reads) endowed with cardiogenic activity.
However, focusing on a single pathway may be somewhat reductionist in view of the multiplicity of interconnected networks that synergistically regulate cell survival, proliferation, differentiation, and function. For this reason, much attention is currently paid to extracellular vesicles as key mediators of these paracrine effects. These vesicles primarily encompass small-sized exosomes $(\leq 150 \mathrm{~nm})$ that originate from cytosolic endosomes and microvesicles (up to $1000 \mathrm{~nm}$ ), which are produced as buds off of the plasma membrane. All these vesicles are rich in biomolecules, particularly noncoding nucleic acids, lipid rafts, and protein fragments, all of which they can shuttle to target cells. Transfer of this payload can result in changes in the transcript profile of the recipient cells, as typically illustrated by the ability of fibroblasts incubated in the presence of cardiosphere-derived microvesicles to exert antifibrotic, anti-apoptotic, and angiogenic effects. ${ }^{7}$ Extracellular vesicles, therefore, are currently considered to be major mediators of intercellular communication, and their ability to turn on pathways that are instrumental in the control of key events, such as neovascularization, cell proliferation, and apoptosis likely accounts for the improved functional outcomes reported after their delivery in animal models of myocardial infarction. ${ }^{8}$

In our laboratory, the functional benefits of transplanting human embryonic stem cell-derived cardiac progenitors in a mouse model of postinfarction chronic heart failure have thus been equaled by the delivery of the sole vesicles collected from those same cells. ${ }^{9}$ Thus, extracellular vesicles quite conceivably may become substitutes for transplantation of the parent cells, provided that their production can be achieved by a scalable and cost-effective purification method, along with the development of standardized and reliable quality controls. Overall, the "pharmaceuticalization" of cellderived biologics would offer the advantages of "off-theshelf" product availability and the elimination of the potential side effects associated with cells, such as uncontrolled proliferation or immune responses. This approach would likely result in greater cost effectiveness, a streamlined regulatory path, and at the end, more clinical applications.

The second important finding from the study by Wei and colleagues ${ }^{1}$ is the potential for biomaterials to foster cardioreparative mechanisms. They used a collagen-made patch, which makes sense both theoretically (collagen is the main component of the extracellular matrix) and practically (medical-grade collagen-based materials are widely available and have a longstanding safety record). However, their collagen was engineered so as to yield an elasticity modulus matching that of the embryonic epicardium $(\sim 12 \mathrm{kPa})$, with the objective of optimizing myocyte contractility.

In practice, materials available for generating cardiac constructs are broadly categorized as follows: natural polymers (such as collagen); synthetic polymers, which are often used in a blended fashion; and decellularized extracellular matrices. All of them have advantages and 
drawbacks. ${ }^{10}$ Natural polymers are biocompatible but may feature variable properties depending on extraction procedures, and they have high production costs. Conversely, synthetic polymers have more reproducible chemical and mechanical properties, which make them better suited for large-scale manufacturing to meet regulatory guidelines for human applications, but their biocompatibility can be hampered by the nature of their degradation products. Decellularized extracellular matrices provide a true scaffolding framework but lack uniform decellularization and introduce variability related to sourcing, processing, and final composition. Scaffold-free cell sheets have the great advantage of biocompatibility, because of the absence of foreign material and promotion of cell survival through their anchoring to a self-produced matrix, but their practical handling can be challenging owing to their frailty.

The study by Wei and colleagues ${ }^{1}$ highlights the fact that beyond the choice of a given material, the method by which it is processed to achieve the best biomimetic properties is a critical factor in determining the ultimate outcome. In this particular case, static compressive stress was applied to obtain an elasticity matching that of the embryonic myocardium, as a means to optimize the function of immature cardiomyocytes. However, the target value of $\sim 12 \mathrm{kPa}$ reported in this study may not be applicable to all settings. For example, in a comparative study of collagen-coated polyacrylamide substrates, Bhana and colleagues ${ }^{11}$ found that the optimal materials for heart cell morphology and function were those with a stiffness comparable to that of the native adult rat myocardium $(22-50 \mathrm{kPa})$; postinfarction myocardial scars feature an even greater stiffness, with elasticity moduli reported to increase by as much as $304 \mathrm{kPa}$ at 6 weeks after myocardial infarction in rat. ${ }^{12}$

The optimal elasticity of cardiac patches thus remains unsettled and likely depends on whether the scaffold is tailored to support the growth and function of the seeded and/or invading host cells, or rather is primarily intended to structurally strengthen the infarcted wall so as to limit its adverse remodeling. Alternatives to the static compression technique are available for generating biomimetic materials. Among these, electrospinning is gaining interest because of its suitability for fine-tuning the 3-dimensional architecture of the scaffold, particularly its surface characteristics, anisotropic fiber alignment, and pore size. The article by Wei and colleagues ${ }^{1}$ appropriately highlights the critical role of these factors in not only providing optimal cues for spatial patterning, survival, and function of the patch-seeded cells, but also guiding cell colonization and vessel ingrowth originating from the host tissue.

A third point, which stems from the previous two, is that the optimal therapeutic strategy might be a combination of factor(s), with an appropriate biomaterial acting as a vehicle. Studies using mouse or rat models of myocardial infarction have shown that the therapeutic efficacy of stem cells could be increased by cotransplanting them with scaffolds functionalized with, respectively, growth factors such as interleukin- $10,{ }^{13}$ or a combination of hepatocyte growth factor and insulin growth factor- $1,{ }^{14}$ to promote retention, engraftment and, consequently, cells' biological effects and their positive impact on heart function.

The concept has even been extended to the mitigation of rejection expectedly triggered by xenogeneic cells, by seeding them onto polymers engineered to sustainably release immunosuppressive agents. ${ }^{15}$ This combination of cell therapy with scaffold-supported growth factors has been clinically tested in the pilot ALCADIA trial (Autologous Human Cardiac-Derived Stem Cell to Treat Ischemic Cardiomyopathy; NCT 00981006), in which intramyocardial injections of autologous cardiac stem cells were combined with placement of a gelatin hydrogel sheet incorporating $\beta$-fibroblast growth factor during a coronary artery bypass procedure. Enhancment of cell function, via combination with such growth factor-loaded materials, has been successfully extended to other areas of regenerative medicine, such as diabetes treatment. ${ }^{16}$

However, the study by Wei and colleagues ${ }^{1}$ goes one step farther by completely eliminating the use of cells and assuming that their effect is (almost) purely factor mediated; it relies exclusively on a factor-loaded polymer to induce reparative effects, which translates into improvement in postinfarction heart function. By itself, the concept is not new. Ten years ago, Hsieh and colleagues ${ }^{17}$ reported on the ability of injectable self-assembling nanofibers embedding insulin growth factor-1 to preserve systolic function and reduce infarct size after myocardial infarction.

Since then, several other growth factors bound to various polymers have been reported to successfully improve heart function after myocardial infarction, and various functionalization strategies have been developed for controlling the temporal and spatial distribution of the delivered factors so as to orchestrate the signaling cues to promote optimally the target pathway activation. ${ }^{18,19}$ For example, mixing 2 polymers that differ by their degradation rate has been performed, with 1 being rapidly released to inhibit neutrophil infiltration, and the other having a longer-term release to recruit circulating hematopoietic stem cells and thereby enhance vascularization of the transplanted area. ${ }^{20}$ The clinical relevance of this approach has been recently demonstrated by successful outcomes after intramuscular administration of $\beta$-fibroblast growth factor sustainably released from gelatin hydrogel microspheres in patients who have chronic limb ischemia. ${ }^{21}$

What these studies share is the delivery of the scaffolded factors via intramyocardial injections. However, injections have several drawbacks, including poor reproducibility (a critical issue for multicenter clinical trials), leakage of the injectate in the pericardium or rapid wash-out through the venous and lymphatic systems, and potential for 


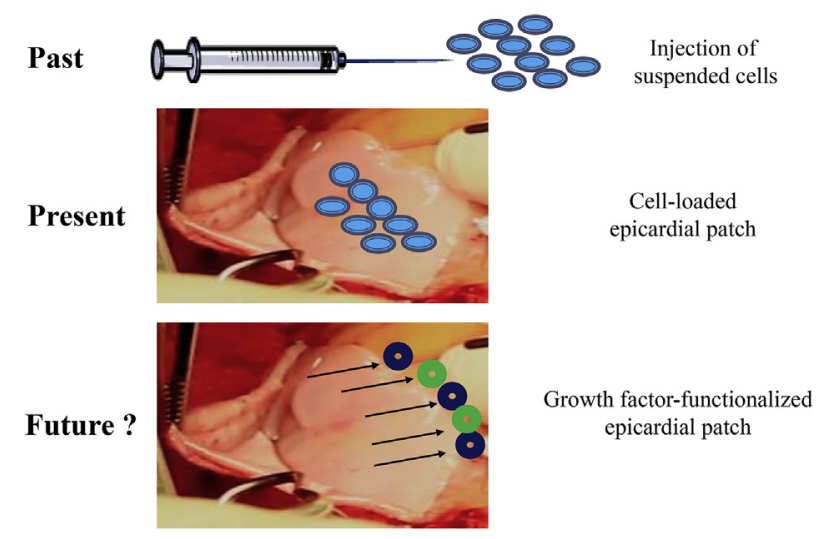

FIGURE 1. Possible evolution of cardiac stem cell therapy.

arrhythmogenesis. Wei and colleagues ${ }^{1}$ therefore have switched, appropriately, from injections to epicardial placement of an Fslt-1-loaded collagen patch. Studies of stem cells have documented that their epicardial delivery as cell-loaded patches is superior to intramyocardial injections. ${ }^{22}$ Based on these results, a reasonable hypothesis is that the same benefits apply to growth factors, ie, their release from reservoir-like biomaterials should enhance local bioavailability, limit off-target effects, and fine-tune the pharmacokinetic profile of the tethered compounds. ${ }^{23}$

However, although Wei and colleagues ${ }^{1}$ chose to bioengineer collagen to deliver Fslt-1, other materials can be considered as long as they can be functionalized, using physical or chemical methods, to incorporate the biological factors within the core of the scaffold or promote their binding at its surface. Because hydrophilic polymers swell and degrade as a result of endogenous water influxes, they may release the embedded factors as uneven bursts, whereas surface-erosion degradable polymers, characteristic of hydrophobic materials, are more suitable for better controlled-release kinetics. The optimal time course of release likely depends on the nature of the factors to be liberated and their tissue targets, but assuming that the released factors reproduce the effects of stem cells, a reasonable speculation is that a roughly 3 -week time frame should be sufficient to induce biological effects, as most stem cells are generally cleared from the transplanted sites by this point.

Finally, the ultimate approach could consist of using biomaterials without stem cells or factors, with the premise that by increasing the thickness of the infarcted wall, they could decrease wall stress on the surviving myocardium and therefore contribute to improved postinfarction function. Although this strategy has yielded encouraging results experimentally, ${ }^{24}$ the mixed functional outcomes of the Randomized, Controlled Study to Evaluate Algisyl-LVR as a Method of Left Ventricular Augmentation for Heart Failure (AUGMENT-HF) trial, in which an alginate hydrogel was intramyocardially injected as a standalone procedure in patients who had advanced heart failure, ${ }^{25}$ call into question the efficacy of using these "unadorned" materials. Rather, a general trend has been found for materials combined with growth factors (or cells) to provide improved benefits, compared with those seen when the materials are delivered alone. ${ }^{26}$ Beyond the specific choice of collagen and Fsit-1, the paper by Wei and colleagues ${ }^{1}$ illustrates clearly the potential interest of these functionalized scaffolds.

In summary, the data of Wei and colleagues ${ }^{1}$ strengthen the concept that in patients who have advanced heart failure, the epicardial placement of a biomimetic material functionalized with factors acting on pathways involved in heart repair (angiogenic growth factors, peptides that can induce mitosis of existing myocytes and/or differentiation and growth of tissue-resident stem cells, regulators of inflammation and fibrosis) may be a promising option (Figure 1). These patches could be used alone or in combination with standard cardiac surgical procedures, eventually by a minimally invasive approach. The relevance of this approach stems from the robustness of the rationale, the consistency of positive experimental data, the potential for industrialscale manufacturing and off-the-shelf storage of the functionalized biomimetic materials, and user-friendly intraoperative implementation.

\section{Conflict of Interest Statement}

Author has nothing to disclose with regard to commercial support.

\section{References}

1. Wei K, Serpooshan V, Hurtado C, Diez-Cuñado M, Zhao M, Maruyama S, et al. Epicardial FSTL1 reconstitution regenerates the adult mammalian heart. Nature. 2015;525:479-85.

2. Masters M, Riley PR. The epicardium signals the way towards heart regeneration. Stem Cell Res. 2014;13(3 Pt B):683-92.

3. Canseco DC, Kimura W, Garg S, Mukherjee S, Bhattacharya S, Abdisalaam S, et al. Human ventricular unloading induces cardiomyocyte proliferation. J Am Coll Cardiol. 2015;65:892-900.

4. Garbern JC, Lee RT. Cardiac stem cell therapy and the promise of heart regeneration. Cell Stem Cell. 2013;12:689-98.

5. Timmers L, Lim SK, Hoefer IE, Arslan F, Lai RC, van Oorschot AA, et al. Mesenchymal stem cell-conditioned medium improves cardiac function following myocardial infarction. Stem Cell Res. 2011;6:206-14.

6. Hynes B, Kumar AH, O'Sullivan J, Klein Buneker C, Leblond AL, Weiss S, et al. Potent endothelial progenitor cell-conditioned media-related anti-apoptotic, cardiotrophic, and pro-angiogenic effects post-myocardial infarction are mediated by insulin-like growth factor-1. Eur Heart J. 2013;34:782-9.

7. Tseliou E, Fouad J, Reich H, Slipczuk L, de Couto G, Aminzadeh M, et al. Fibroblasts rendered antifibrotic, antiapoptotic, and angiogenic by priming with cardiosphere-derived extracellular membrane vesicles. J Am Coll Cardiol. 2015;66:599-611.

8. Cervio E, Barile L, Moccetti T, Vassalli G. Exosomes for intramyocardial intercellular communication. Stem Cells Int. 2015;2015:482171.

9. Kervadec A, Bellamy V, El Harane N, Arakélian L, Vanneaux V, Cacciapuoti I, et al. Cardiovascular progenitor-derived extracellular vesicles recapitulate the beneficial effects of their parent cells in the treatment of chronic heart failure. J Heart Lung Transplant. 2016;35:795-807.

10. Tallawi M, Rosellini E, Barbani N, Cascone MG, Rai R, Saint-Pierre G, et al. Strategies for the chemical and biological functionalization of scaffolds for cardiac tissue engineering: a review. J R Soc Interface. 2015;12:20150254. 
11. Bhana B, Iyer RK, Chen WL, Zhao R, Sider KL, Likhitpanichkul M, et al. Influence of substrate stiffness on the phenotype of heart cells. Biotechnol Bioeng. 2010; 105:1148-60.

12. Atluri P, Trubelja A, Fairman AS, Hsiao P, MacArthur JW, Cohen JE, et al. Normalization of postinfarct biomechanics using a novel tissue-engineered angiogenic construct. Circulation. 2013;128(11 Suppl 1):S95-104.

13. Holladay CA, Duffy AM, Chen X, Sefton MV, O'Brien TD, Pandit AS. Recovery of cardiac function mediated by MSC and interleukin-10 plasmid functionalised scaffold. Biomaterials. 2012;33:1303-14.

14. Savi M, Bocchi L, Fiumana E, Karam JP, Frati C, Bonafé F, et al. Enhanced engraftment and repairing ability of human adipose-derived stem cells, conveyed by pharmacologically active microcarriers continuously releasing HGF and IGF1, in healing myocardial infarction in rats. J Biomed Mater Res A. 2015;103: 3012-25.

15. Song TH, Jang J, Choi YJ, Shim JH, Cho DW. 3D-printed drug/cell carrier enabling effective release of cyclosporin A for xenogeneic cell-based therapy. Cell Transplant. 2015;24:2513-25.

16. Vernon RB, Preisinger A, Gooden MD, D’Amico LA, Yue BB, Bollyky PL, et al. Reversal of diabetes in mice with a bioengineered islet implant incorporating a type I collagen hydrogel and sustained release of vascular endothelial growth factor. Cell Transplant. 2012;21:2099-110.

17. Hsieh PC, Davis ME, Gannon J, MacGillivray C, Lee RT. Controlled delivery of PDGF-BB for myocardial protection using injectable self-assembling peptide nanofibers. J Clin Invest. 2006;116:237-48.

18. Segers VF, Lee RT. Biomaterials to enhance stem cell function in the heart. Circ Res. 2011;109:910-22.

19. Formiga FR, Pelacho B, Garbayo E, Imbuluzqueta I, Díaz-Herráez P, Abizanda G, et al. Controlled delivery of fibroblast growth factor-1 and neuregulin-1 from biodegradable microparticles promotes cardiac repair in a rat myocardial infarction model through activation of endogenous regeneration. J Contr Release. 2014;173:132-9.
20. Projahn D, Simsekyilmaz S, Singh S, Kanzler I, Kramp BK, Langer M, et al Controlled intramyocardial release of engineered chemokines by biodegradable hydrogels as a treatment approach of myocardial infarction. J Cell Mol Med. 2014;18:790-800.

21. Kumagai M, Marui A, Tabata Y, Takeda T, Yamamoto M, Yonezawa A, et al Safety and efficacy of sustained release of basic fibroblast growth factor using gelatin hydrogel in patients with critical limb ischemia. Heart Vessels. April 11, 2015 [Epub ahead of print].

22. Hamdi H, Furuta A, Bellamy V, Bel A, Puymirat E, Peyrard S, et al. Cell delivery: intramyocardial injections or epicardial deposition? A head-to-head comparison. Ann Thorac Surg. 2009;87:1196-203.

23. Fathi E, Nassiri SM, Atyabi N, Ahmadi SH, Imani M, Farahzadi R, et al. Induction of angiogenesis via topical delivery of basic-fibroblast growth factor from polyvinyl alcohol-dextran blend hydrogel in an ovine model of acute myocardial infarction. J Tissue Eng Regen Med. 2013;7:697-707.

24. Tous E, Ifkovits JL, Koomalsingh KJ, Shuto T, Soeda T, Kondo N, et al Influence of injectable hyaluronic acid hydrogel degradation behavior on infarction-induced ventricular remodeling. Biomacromolecules. 2011; $12: 4127-35$

25. Mann DL, Lee RJ, Coats AJ, Neagoe G, Dragomir D, Pusineri E, et al. One-year follow-up results from AUGMENT-HF: a multicentre randomized controlled clinical trial of the efficacy of left ventricular augmentation with Algisyl in the treatment of heart failure. Eur J Heart Fail. November 11, 2015 [Epub ahead of print].

26. Nelson DM, Ma Z, Fujimoto KL, Hashizume R, Wagner WR. Intra-myocardia biomaterial injection therapy in the treatment of heart failure: materials, outcomes and challenges. Acta Biomater. 2011;7:1-15.

Key Words: heart failure, biomaterials, stem cells, paracrine signaling effects by 